\title{
The effects of body size and vitamin $D$ status on bone mass of British women from South Asian and European origin: results from the Vitamin D, Food Intake, Nutrition and Exposure to Sunlight in Southern England (D-FINES) Study
}

\author{
W. T. K. Lee, A. L. Darling, P. A. Lee, A. Osborn, F. Hanjra, P. Duckworth, P. Cardew, S. Patel, \\ R. Gray and S. A. Lanham-New \\ Faculty of Health and Medical Sciences, University of Surrey, Guildford GU2 7XH, UK
}

There are limited studies on factors determining bone mineral density (BMD) in South Asians (SA) women of UK origin. BMD of SA women is found to be lower than that of Caucasians (Cau), but often this difference has been confounded in a number of studies by body size $\mathrm{e}^{(1,2)}$. The aim was to examine factors modulating variation in BMD, bone mineral content (BMC) and bone area (BA) among $\mathrm{SA}$ and Cau women of UK origin.

The data are derived from the D-FINES Study (2006-7) examining the effect of vitamin D status on bone health of women living in Surrey, UK. In the present study Autumn (September-December 2006) data from 185 premenopausal women (Cau: $n$ 135, age 33.9 (SD 5.6) years; SA: $n$ 50, age 38.0 (SD 8.9) years) were examined. Bone mass and body composition were measured by dual X-ray absorptiometry (DXA; Hologic QDR; Hologic UK Ltd, Crawley, West Sussex, UK). Other measurements taken included dietary intake, physical activity (PA) level, hand-grip strength and serum concentrations of 25-hydroxyvitamin D (25OHD) and parathyroid hormone (PTH).

Trochanter BMD (TroBMD), spinal BMC (LSBMC), spinal bone area (LSBA), total body BMD (TBMD), total body BMC (TBMC) of SA were all significantly lower than those of Cau $(P<0.025)$. Furthermore, SA had significantly lower height, hand-grip strength, PA level and serum 25OHD than Cau $(P \leq 0.004)$. However, PTH of SA was significantly higher than that of Cau $(P<0.0001)$. Dietary Ca and vitamin D intake did not differ between SA and Cau. Univariate correlation between bone mass measures and other variables was determined. Variables with $P<0.05$, i.e. age, weight, height, BA, serum $25 \mathrm{OHD}$ and PTH, hand-grip strength, PA level, total fat mass (TFM; by DXA) and menarche age were then entered into the regression models to determine their relative contribution to the variation in bone mass.

Ethnic differences in TroBMD, LSBMC, LSBA, TBMD and TBMC were not found when adjustment was made for age, weight and height in the initial regression models. For TroBMD, weight $(P=0.04)$, BA $(P<0.0001)$ and serum $25 \mathrm{OHD}(P=0.033)$ remained significant determinants in the final model (adjusted $\left.R^{2} 45 \%\right)$. For LSBMC, weight $(P=0.006)$ and LSBA $(P<0.0001)$ were significant determinants in the initial model (adjusted $\left.R^{2} 67.6 \%\right)$. However, weight was not significant when 25OHD $(P=0.022)$ entered the model (adjusted $\left.R^{2} 68 \%\right)$. For TBMC, weight and height $(P<0.0001)$, 25OHD $(P=0.035)$, and TFM $(P=0.003)$ remained significant in the final model to predict TBMC variation (adjusted $\left.R^{2} 60.7 \%\right)$. For TBMD, weight $(P=0.001), 25 \mathrm{OHD}(P=0.018)$ and TFM $(P=0.032)$ were significant determinants of TBMD after adjusting for other covariates (adjusted $R^{2} 31 \%$ ).

These findings suggest that lower bone mass among premenopausal SA women living in England was associated with their smaller body size. Body weight was found to be a consistent determinant of bone mass in various skeletal sites after adjusting for confounding factors. It is important to note that serum vitamin D status was a very significant determinant of the variation in bone mass even after controlling for ethnicity, body size, bone size and other confounding factors. In addition, TFM was also found positively associated with bone mass. SA women are at greater risk of osteoporosis because of their smaller body size, darker skin colour (less cutaneous vitamin D synthesis), lower serum 25OHD concentration and physically-inactive lifestyle. Promotion of higher peak bone mass in younger SA women by promoting a healthy body weight, regular sunshine exposure, regular PA and the use of vitamin D-fortified foods will be important to prevent osteoporosis in later life.

The D-FINES Study is funded by the FSA (Project no. 5064).

1. Roy D, Swarbrick C, King Y et al. (2005) Osteoporos Int 16, 1254-1262.

2. Ward KA, Roy DK, Pye SR et al. (2007) Bone 41, 117-121. 